\title{
Critical Care Management and Newer Therapies for Tumour Lysis Syndrome
}

\author{
Uma Hariharan* and Vinodh Natarajan
}

Ram Manohar Lohia Hospital and Post Graduate Institute of Medical Education and Research, Central Health Services, New Delhi, India

\begin{abstract}
Cancer disorders are the one of the leading causes of death in most developed and developing countries. They constitute a diverse group of pathologies in which abnormal metabolism and life cycle lead to the profound derangement's of host metabolism. With the recent advancement in the medical field, many cancer diseases are well controlled with early diagnosis and treatment. So, it is essential that clinicians must be aware the complications of cancer itself and its management. Tumour lysis syndrome (TLS) is an onco-metabolic emergency which occurs due to cancer cells targeted treatment (chemotherapy or radiotherapy) or from rapidly dividing cancer cells itself (spontaneous TLS). TLS was first described by Bedrna and Polcak in 1929 in a chronic leukemia patient treated with radiotherapy. It is characterized by a group of metabolic derangements characterised by hyperuricemia, hyperkalemia, hyperphosphatemia, hypocalcemia and uremia. The consequences are potentially lethal and may result in acute kidney failure, seizures, tetany and even sudden death. The scenario is further complicated in patient with underlying comorbid conditions and in patients at extremes of ages.
\end{abstract}

\section{Introduction}

\section{Pathophysiology}

Tumour lysis syndrome is an oncologic emergency. With increase in the detection of various cancers and the advent of newer chemotherapeutic agents, there are greater chances of increases in the incidence of cancer chemotherapy-related complications, including TLS. Massive and sudden release of cell-breakdown products into the circulation triggers a multitude of systemic effects, leading to renal shut down, dys-electrolytemias, hyperuricemia, hypovolemia and cardiac arrhythmias. The Cairo and Bishop Classification system 2004 is commonly used for categorising TLS into two main types $[1,2]$ on the basis of specific laboratory (L-TLS) and clinical features (C-TLS). The latter type generally requires admission to the ICU.

\section{Risk Stratification and Review of Literature}

Incidence of tumour lysis syndrome is about $3-12 \%$ among the high grade hematological malignancies and was observed in less than $0.5 \%$ of targeted chemotherapy for haematological malignancy. There were few case reports in the literature of tumour lysis syndrome associated with solid malignancies. Certain intrinsic factors like old age, pre-existing renal problems, oliguria, dehydration, elevated baseline uric acid levels and rapid cytoreductive therapy may predispose to the development of tumour lysis syndrome [3].

Whenever assessing the risk of TLS, it is always important to keep in mind both the general and tumour related predictors of risk. An older age is associated with reduction in glomerular filtration rate and may complicate volume replacement therapy due to the higher rates of cardiac dysfunction. Patients afflicted with cancer often have decreased oral intake due to loss of appetite and nausea. Furthermore, the cancer patients often suffer from vomiting and they might use medications like NSAIDs (Non-steroidal Anti-inflammatory Agents) capable of detrimentally affecting renal function. The medication list of the cancer patient should be reviewed and nephrotoxic drugs should be stopped wherever possible. Other general systemic disease like cardiac disease, diabetes mellitus and renal disease should be considered prior to hydration, as these people can easily develop symptomatic volume overload.
Another aspect of risk stratification is the type and burden of malignancy. Based on that, there are three types of risks. High risk group of cancers include advanced Burkitt lymphoma/leukemias (lactate dehydrogenase levels elevated to twice the upper limit of normal (ULN)), acute lymphocytic leukemia (ALL) with WBC count $>100000$, acute myeloid leukemia (AML) with WBC count $>100000$ and diffuse large B cell lymphoma with elevated baseline LDH levels of twice upper normal limit, and bulky disease. Intermediate risk group includes AML with WBC count between 25000 and 100000, ALL with WBC $<100000$ and LDH less than twice of the ULN, early stage Burkitt lymphoma/leukemia with LDH of less than ULN, and diffuse large B-cell lymphoma with baseline increase in LDH of twice ULN but nonbulky disease. Low risk malignancies include indolent lymphomas, chronic lymphocytic lymphomas, chronic myeloid leukemia in chronic phase, AML with WBC $<25000$, multiple myeloma and solid cancers [4]. Extra attention should be paid to patients with Burkitt lymphomas/ leukemias, ALL, AML, diffuse large B-cell lymphomas [5], tumours highly sensitive to therapy and small cell lung cancers. Both tumours related and general patient related factors should be included in the risk stratification. Certain patient factors like advanced age and the presence pre-existing renal and cardiac problems warrant a closer follow up during the preventive hydration therapy. Algrin et al. [5] did a retrospective analysis on 190 patients admitted in ICU and found that the early admission of high risk patients will be helpful in the early recognition of organ failure and complications so that appropriate treatment can be given at the right time. The author also quoted that ICU admission of high risk patients may improve the overall one-year

*Corresponding author: Hariharan U, Ram Manohar Lohia Hospital and Post Graduate Institute of Medical Education and Research, Central Health Services, New Delhi, India, Tel: 9811271093; E-mail: uma1708@gmail.com

Received October 30, 2017; Accepted November 15, 2017; Published November 25, 2017

Citation: Hariharan U, Natarajan V (2017) Critical Care Management and Newer Therapies for Tumour Lysis Syndrome. J Blood Lymph 7: 189. doi: 10.4172/21657831.1000189

Copyright: (c) 2017 Hariharan U, et al. This is an open-access article distributed under the terms of the Creative Commons Attribution License, which permits unrestricted use, distribution, and reproduction in any medium, provided the original author and source are credited. 
survival rates. Lenglin et al. [6] in 2010 compared early ICU versus late ICU admission of haematological malignancy patient undergoing chemotherapy. The investigator found that patients admitted late in the ICU had higher incidence of non-invasive ventilation failures and acute renal insufficiency which further led to increased morbidity and mortality rates in them [7]. Many other studies also quoted the induction of chemotherapy can be done safely in the intensive care setting for high risk and intermediate risk patients, as well as patients with underlying renal and cardiac problems. Coiffier et al. [8] in their study recommended that patients should be hydrated to maintain urine output of at least $2 \mathrm{ml} / \mathrm{kg} / \mathrm{h}$. to minimize the risk of acute kidney injury. Hence, it is clear that intensivists play a vital role in the management of haematological malignancy patient undergoing cancer cell targeted therapy.

\section{Before admission in ICU}

After the proper discussion with haematologists, the highrisk group patient gets early admission in ICU for initiation of chemotherapy. Proper history, underlying medical problems, any other past relevant history should be noted before admission. The medication list of the patient should be reviewed before the admission in ICU. Before shifting the patient to ICU, it is mandatory to have all the baseline investigations like complete blood count, renal function test, liver function test, serum electrolytes including serum phosphorous and uric acid levels, chest X-ray, coagulation profile and 12-lead electrocardiogram (ECG). Screening echocardiography might be useful to assess the left ventricular function and to find out any regional wall motion abnormalities before initiating chemotherapy. As these patients are prone to get infected easily, proper isolation of bed in ICU and all strict aseptic precautions should be carried out effectively during all procedures [3].

\section{Management in ICU}

It is essential to keep in mind that prevention of disease is always more cost effective and safer than the treatment of an established disease. Recognition of risk factors, close monitoring of at-risk patients and timely interventions are the key to preventing or managing tumour lysis syndrome [4].

Before initiating chemotherapy or radiotherapy in ICU, all the patient should be connected to basic ASA (American Society of Anesthesiologists) standard monitoring like ECG (especially lateral leads), $\mathrm{SpO}_{2}$, heart rate and non-invasive blood pressure measurement. Airway patency should be checked and the need for invasive ventilation should be assessed with baseline arterial blood analysis. Most of the patients do not require endotracheal intubation if admitted early to ICU and can be managed with supplemental oxygen supply alone. Patients with underlying cardiac or renal problems should have invasive arterial blood pressure measurement to assess the hemodynamic changes and blood gases, under strict aseptic precautions after performing Allen's test. Central line placement is beneficial before initiating chemo or radiotherapy, so that volume status of the patient can be monitored throughout the procedure and emergency drugs can be infused easily. In the event of a renal replacement therapy in future, central line can be easily converted to permacath (dialysis catheter) with use of appropriate sized guide wire. Central line placement should be done preferably under ultrasound guidance, as these patients might have low platelet count and deranged coagulation profile.

Patient management should be focussed on the basis of the type of cancer and certain biochemical parameters such as serum LDH, phosphorous, uric acid, potassium, calcium and serum creatinine levels. Adequate hydration of patient, use of allopurinol/rasburicase (uric acid decreasing agents) and phosphate binders is the mainstay of treatment for the prevention of TLS in patients undergoing active therapy [9]. The choice of fluid varies and some recommend the use of dextrose with one quarter normal saline in it. Normal saline or ringer lactated solution are other choices, but it is essential to remember that ringer lactated solution contains potassium (aggravating hyperkalemia) and the use of normal saline may lead to hyperchloremic metabolic acidosis. Patients with underlying cardiorenal disease may suffer from over-hydration and risk of developing fluid overload and pulmonary edema. Use of loop diuretics in such situations might be helpful. Those patients at intermediate risk of developing TLS should receive allopurinol at least 48 or $24 \mathrm{~h}$ prior to the initiation of targeted cell therapy to prevent the risk of uric acid nephropathy. Patients, who cannot tolerate the oral medications such as those with nausea or vomiting and altered gastrointestinal functions, receive allopurinol IV. The recommended dose of allopurinol is $800 \mathrm{mg}$ orally or $600 \mathrm{mg}$ IV daily and should be continued for 4-7 days. Allopurinol works by blocking xanthine oxidase which is a key enzyme in purine catabolism. Allopurinol, in rare instances is associated with hypersensitivity reactions and may lead to acute kidney injury in the form of acute interstitial nephritis. Dosage reduction should be done in the setting of chronic kidney disease. So, in such situations, use of another new xanthine oxidase inhibitor, Febuxostat is recommended [10-13]. Nevertheless, despite the availability of allopurinol, there is significant number of patient who still develop kidney damage due to uric acid crystals. Some mammals but not humans possess the uricase enzyme which is capable of converting xanthine into Allantoin (easily excreted in urine). A recombinant form of uricase enzyme, Rasburicase is available and is effective in reducing the level of uric acid, serum creatine and other metabolites responsible in developing TLS. A recent metanalysis done by Feng et al. suggested that a single dose of Rasburicase will be effective in controlling the levels of uric acid when combined with Allopurinol [11]. Cortes et al. demonstrated in his study that Rasburicase provides better control of uric acid levels than allopurinol [13]. FDA recommends the dosing of $0.15-0.2 \mathrm{mg} / \mathrm{kg}$ once daily in $50 \mathrm{~mL}$ of normal saline as an IV infusion for 5 days. Uric acid levels should be monitored regularly and treatment can be stopped if uric acid levels are lower or not detectable [1]. Despite being a safe agent, Rasburicase should not be used in pregnant patients and patients with glucose-6-phosphate dehydrogenase deficiency due to risk of hemolysis and methemoglobinemia [13].

Historically, alkalinisation of urine had been recommended for pediatric patients receiving the treatment for hyperuricemia to promote excretion of uric acid. However, this practice is not recommended nowadays, due to risk of precipitation of calcium phosphate crystals [14]. The use of phosphate binders in the prevention of TLS requires further randomised controlled trials. The decision to start phosphate binders should be based on individual case and most commonly used phosphate binders are calcium salts. Certain parameters should be monitored in patients at risk of developing TLS such as uric acid, potassium, phosphorous and calcium after $4 \mathrm{~h}$ of initiation of chemotherapy or radiotherapy. The discontinuation of prophylaxis should be considered after the completion of cancer-related treatment when serum markers (uric acid, phosphorous, calcium and potassium) for at least two consecutive measurement several hours apart. It is reasonable to monitor the patient in ICU for at least $24 \mathrm{~h}$ after discontinuation of TLS prophylaxis, to ensure no development of TLS [4].

The management of full blown TLS is based on same principles as that of its prevention. As mentioned earlier, the clinical spectrum 
of TLS includes the laboratory abnormalities such as hyperkalemia, hyperphosphatemia, hyperuricemia and hypocalcemia, which present clinically as cardiac arrhythmias, tetany, seizures and acute kidney injury. Hyperkalemia is a dangerous entity which may lead to muscle fatigue, cardiac rhythm disturbances and sudden cardiac arrest. Management of hyperkalemia always start with 12 lead electrocardiograms. Calcium chloride or carbonate salts are used for stabilizing cardiac activity and reserved for malignant cardiac arrhythmias. Insulin glucose infusion should be started as soon as possible for the fast correction of hyperkalemia. If blood sugar is above $200 \mathrm{mg} / \mathrm{dL}$, dextrose containing solutions can be avoided. Salbutamol/Albuterol nebulisation (beta2 agonist) can be added up to patient treatment schedule. Another way of lowering potassium levels is by the use of sodium polystyrene sulfonate resins. Sodium polystyrene sulfonate acts in the intestine by combining with potassium in exchange with sodium. It can be administered orally or as enema and starts effective action after $2 \mathrm{~h}$ and acts for the duration of 6-8 h. However, refractory hyperkalemia should be treated with renal replacement therapy [15]. Elevated uric acid levels are managed with Rasburicase once or twice daily dose. Allopurinol can be used if Rasburicase is contraindicated or unavailable. Use of allopurinol itself can damage kidney function and in such patient hemodialysis will be helpful. Management of elevated phosphorous levels in TLS patient includes restriction of phosphate intake in both diet and IV fluids. Phosphate binders can be used in the setting of full blown TLS and non-calcium salt binders (Sevelamer and Lanthanum) are more preferred than calcium salts. They can be taken with each diet and works by reducing the intestinal absorption of phosphorous. Important consideration should be given to TLS patients with hypocalcemia. Usually, hypocalcemia-associated with TLS should not be treated with calcium supplementation due to risk of calcium phosphate formation and organ injury [16]. It should be reserved for malignant cardiac arrhythmias (VT/VF), cardiac arrest, and tetany. Hemodialysis or renal replacement therapy should be considered for refractory hyperphosphatemia, symptomatic hypocalcemia and calcium phosphate product $>70 \mathrm{mg}^{2} / \mathrm{dL}^{2}$. In the cardiac arrest situation, it is mandatory to follow recent advanced cardiac life support (ACLS) 2015 guidelines for the management and to exclude the other possible causes of cardiac arrest such as hyperkalemia, hypokalemia, hypovolemia, acidosis, cardiac tamponade, pulmonary or coronary thrombosis and toxin exposure [17].

\section{Conclusion}

Tumour lysis syndrome is an onco-metabolic emergency situation requiring meticulous care and management. The complications can be well prevented by maintaining adequate hydration, use of uric acid lowering agents, phosphate binders and minimization of potassium intake. It is important to identify at risk patients and shift them early to ICU for proper management, vigilant monitoring and prevention of organ failure.

\section{References}

1. Jones GL, Will A, Jackson GH, Webb NJ, Rule S, et al. (2015) Guidelines for the management of tumour lysis syndrome in adults and children with haematological malignancies on behalf of the British Committee for Standards in Haematology. Br J Haematol 169: 661-671.

2. Cairo MS, Bishop M (2004) Tumour lysis syndrome: new therapeutic strategies and classification. Br J Haematol 127: 3-11.

3. Cairo MS, Coiffier B, Reiter A, Younes A, Expert TLS (2010) Recommendations for the evaluation of risk and prophylaxis of tumour lysis syndrome (TLS) in adults and children with malignant diseases: an expert TLS panel consensus. Br J Haematol 149: 578-586.

4. Mirrakhimov AE, Ali AM, Khan M, Barbaryan A (2014) Tumor Lysis Syndrome in Solid Tumors: An up to Date Review of the Literature. Rare Tumors 6: 5389.

5. Algrin C, Faguer S, Lemiale V, Lengliné E, Boutboul D, et al. (2015) Outcomes after intensive care unit admission of patients with newly diagnosed lymphoma. Leuk Lymphoma 56: 1240-1245.

6. Lengliné E, Raffoux E, Lemiale V, Darmon M, Canet E, et al. (2012) Intensive care unit management of patients with newly diagnosed acute myeloid leukemia with no organ failure. Leuk Lymphoma 53: 1352-1359.

7. Ayed S, Bornstain C, Vincent F (2016) Evolving concepts in tumour lysis syndrome management. Br J Haematol 173: 485-486.

8. Coiffier B, Altman A, Pui CH, Younes A, Cairo MS (2008) Guidelines for the management of pediatric and adult tumor lysis syndrome: an evidence-based review. J Clin Oncol 26: 2767-2778.

9. Keenan RT (2012) Safety of urate-lowering therapies: managing the risks to gain the benefits. Rheum Dis Clin North Am 38: 663-680.

10. Takai M, Yamauchi T, Fujita K, Shin L, Ookura M, et al. (2014) Controlling serum uric acid using febuxostat in cancer patients at risk of tumor lysis syndrome. Oncology Letters 8: 1523-1527.

11. Feng X, Dong K, Pham D, Pence S, Inciardi J, et al. (2013) Efficacy and cost of single-dose rasburicase in prevention and treatment of adult tumour lysis syndrome: a meta-analysis. J Clin Pharm Ther 38: 301-308.

12. Lopez-Olivo MA, Pratt G, Palla SL, Salahudeen A (2013) Rasburicase in tumo lysis syndrome of the adult: a systematic review and meta-analysis. Am J Kidney Dis 62: 481-492.

13. Cortes J, Moore JO, Maziarz RT, Wetzler M, Craig M, et al. (2010) Contro of plasma uric acid in adults at risk for tumor Lysis syndrome: efficacy and safety of rasburicase alone and rasburicase followed by allopurinol compared with allopurinol alone--results of a multicenter phase III study. J Clin Oncol 28 : 4207-4213.

14. Worcester EM, Coe FL (2010) Clinical practice: Calcium kidney stones. N Eng J Med 363: 954-963.

15. Maxwell AP, Linden K, O'Donnell S, Hamilton PK, McVeigh GE (2013) Management of hyperkalaemia. J R Coll Physicians Edinb 43: 246-251.

16. Habbous S, Przech S, Acedillo R, Sarma S, Garg AX et al. (2017) The efficacy and safety of sevelamer and lanthanum versus calcium-containing and ironbased binders in treating hyperphosphatemia in patients with chronic kidney disease: a systematic review and meta-analysis. Nephrol Dial Transplant 32 111-125.

17. Link MS, Berkow LC, Kudenchuk PJ, Halperin HR, Hess EP, et al. (2015) Part 7: Adult Advanced Cardiovascular Life Support: 2015 American Heart Association Guidelines Update for Cardiopulmonary Resuscitation and Emergency Cardiovascular Care. Circulation 132: S444-S464. 